\title{
Research of Sensor Fault Detection and Diagnosis for EMB System Based on CSA-SVM Model
}

\author{
Z. J. Yu and Y. N. Xu
}

\begin{abstract}
Electro Mechanical Brake (EMB) is a high efficiency pure electric vehicle braking system which is based on the technology of Electronic, machinery, communication network. Because of the expensive cost and immature key technology in safety and reliability, the products cannot be mass-produced on the market at present. Electronic control of the EMB system needs a variety of sensors information feedback, therefore, how to correctly detect and diagnose the faults of the sensors is one of the important problems for the development of pure electric vehicles. Based on three-loop control architecture model of EMB system, the sensor fault detection model is established on the basis of Support Vector Regression (SVR), and the sensor fault diagnosis model is established on the basis of Support Vector Classification (SVC). In order to further improve the Support Vector Machine (SVM) for fault classification accuracy and fault detection reliability, the parameters of SVM can be optimized by using Clonal Selection Algorithm (CSA) and the modified CSA-SVM model is established. Simulated result of experiment indicates that the proposed CSA - SVM fault detection rate is increased than the traditional SVM by $62.5 \%$ and the fault classification accuracy is increased by $10 \%$ which laids a solid foundation of fault-tolerant control technology for the EMB system.
\end{abstract}

Index Terms-Fault detection, fault diagnosis, support vector machine, clonal selection, electro mechanical brake.

\section{INTRODUCTION}

Automotive braking system can directly affect the safety of the car. EMB system is a gordian technique to fundamentally improve the automobile braking performance, steering stability, intelligent degree and economic performance of the energy consumption [1]. Along with the automobile intelligent and electrification process continues to accelerate, the related technology continues to mature, not only making EMB system based on the technology of electromechanical integration and drive-by-wire possible, but also becoming the development direction of the next generation of pure electric vehicle braking system. The EMB system over the traditional hydraulic braking system has the advantages of mall size, high efficiency, simple structure, convenient installation and maintenance, flexible layout and so on. It also has the active adaptation to different drivers under different driving conditions. The electronic brake pedal simulator and actuators of EMB system is carrying with

Manuscript received June 7, 2014; revised July 30, 2014. This work was supported by the National Natural Science Foundation of China (61361003) and Jilin University of State Key Laboratory of Automotive Simulation and Control open fund (20121120)

The authors are with the division of electronic and communication engineering of Yanbian University, Yanji, China (e-mail: 2013050243@ybu.edu.cn,ynxu*@ybu.edu.cn). current sensor, speed sensor and so on [2]. In electronic control system, sensor is a very important approach and methods to perceive, test and obtain the information. Due to sensor will be directly affected by the electromagnetic field. At the same time, except car outside influences such as vibration and temperature, it is also affected by the electronic components own life. There will be a sensor fault such as constant gain, constant deviation and stuck faults, etc. Once the sensor broke down, the driver's breaking intention and braking effect could not be accurately detected, it will brings a serious threat to the braking system. Therefore, the fault detection and diagnosis technology of the sensor are particularly necessary and important.

A robust fault detection filter which is effective for the sensor fault diagnose is proposed in [3]. The method can effectively detect the sensor fault, however, this method is put forward for the linear system, it cannot adapt to the complex, integration problem in nonlinear systems. There is a method using BP neural network method to the research of sensor fault diagnosis in [4]. In [5], a fault diagnosis is put forward for temperature, flow rate and pressure sensors in VAV systems using wavelet neural network. It realizes the fault diagnosis for sensors by using neural network algorithm which has the function of self-learning, parallel processing and fault tolerance. But under the condition of small sample, over fitting phenomenon will easily arise leading to the low generalization ability. In addition, the neural network algorithm is greatly influenced by topology structure complexity of network and the data complexity. These deficiencies making the application effect of neural network algorithm in fault diagnose technology is much less than the desired effect. One sensor fault diagnose method is proposed based on support vector regression algorithm in [6]. Support vector regression is a new machine learning algorithm based on statistical learning theory which has very strong capability to handle the complicated nonlinear system. Support Vector Machine can solve the small-sample problems well by using the structural risk minimization principle. It has the advantages such as the only global optimal solution, strong generalization ability and the structure of the model determining by the algorithm to automatically determine. But the performance of support vector machine is depended on the problems of parameters selecting. Parameter selection based on prior knowledge and the regression model are hard to reach the targets of intelligent fault detection and fault-tolerant control of nonlinear systems. From the above analysis study, the traditional sensor fault diagnosis technology cannot meet the requirements of nonlinear systems, as well as the neural network algorithm for nonlinear system has the shortcomings such as a badly 
affected by the sample, parameter selection, which is difficult to achieve accurate fault detection and diagnosis.

For EMB system, a sensor fault detection and diagnose model based on SVM is put forward, it is based on clonal selection algorithm aiming at parameters selection, and the support vector machine is applied to EMB system. In section II, the three-loop control architecture model of EMB is constructed. Section III sets up the fault detection and diagnosis model based on SVM. Section IV presents a SVM parameter optimization algorithm based on CSA. A sensors fault detection and diagnose model based on modified CSA-SVM has been established in section V and then the simulation using MATLAB is given in Section VI. Finally, brief conclusions are given in section VII. Simulation results show that the modified CSA-SVM model can detect and diagnose faults steadily and effectively, and laid a solid foundation to the security and reliability of the EMB system.

\section{The ThreE-LoOP CONTROL OF EMB SYSTEM ARCHITECTURE MODEL}

EMB system mainly includes controller, actuator and sensor system. EMB actuators are mainly composed of retarding mechanism, movement conversion device, and executive motor [7]. Where, EMB system pressure control, speed control and current control loop can be realized by the action of executive motor current, speed and braking pressure signal, such as shown in Fig. 1.

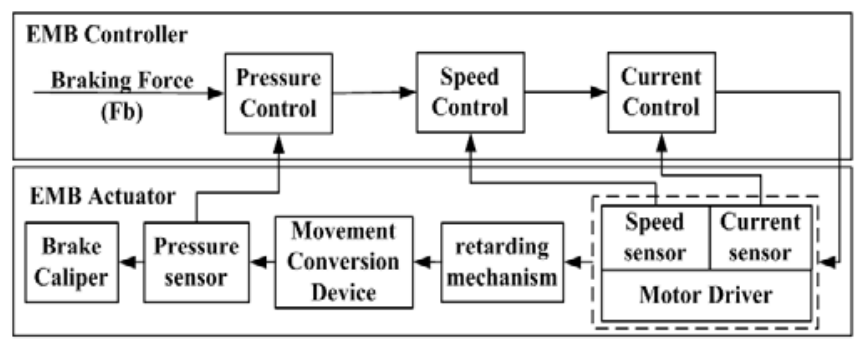

Fig.1. Three-loop control of EMB system architecture model.

EMB system whether run normally or not depends largely on the accuracy of the sensor signal such as the current, speed, pressure, etc. Where, the current and speed signal are from the EMB system of Brushless Direct Current Motor. Through the establishment of the brushless motor mathematical model to acquire the current and speed sensor output model, to establish the output of the pressure sensor model according to the relationship between the braking force and the load torque.

When the EMB system of brushless motor is working normally, it can be described by using the following equation [8].

$$
\begin{gathered}
L_{a} \frac{d I_{a}}{d t}+R_{a} I_{a}+E_{b}=E_{a} \\
E_{b}=k_{b} \dot{\omega}_{m} \\
J_{m} \ddot{\omega}_{m}+B_{m} \dot{\omega}_{m}=T_{m}-T_{f} \\
T_{m}=k I_{a}
\end{gathered}
$$

where, $E_{a}$ is armature voltage, $E_{b}$ is armature counter electromotive force, $I_{a}$ is armature current, $L_{a}$ is the armature inductance, $k$ is moment coefficient, $k_{b}$ is feedback coefficient of electromotive force, $R_{a}$ is armature resistance, $J_{m}$ is equivalent moment of inertia of the motor shaft, $\omega_{m}$ is corner, $T_{m}$ is motor torque, $T_{f}$ is motor load torque, $B_{m}$ is motor shaft equivalent viscous friction coefficient.

According to the equation (1)-(4), the state space expression of brushless motor model can be concluded as follows.

$$
\dot{x}=\left[\begin{array}{cc}
-\frac{R_{a}}{L_{a}} & -\frac{k_{b}}{L_{a}} \\
\frac{k}{J_{m}} & -\frac{B_{m}}{J_{m}}
\end{array}\right] x+\left[\begin{array}{cc}
\frac{1}{L_{a}} & 0 \\
0 & -\frac{1}{J_{m}}
\end{array}\right] u \text {. }
$$

where, $x=\left[\begin{array}{c}I_{d} \\ \dot{\omega}_{m}\end{array}\right]$.

According to the relationship between the structure of EMB actuators, braking force and the motor load torque, the relational expression is $T_{L}=\eta \dot{F}_{b}$. Where, $\dot{F}_{b}$ is the braking force, $\eta$ is the brake factor.

\section{SEnSOr FAult Detection AND Diagnosis Model BASED ON SUPPORT VECTOR MACHINE}

SVM is a powerful learn method machine which is proposed by V. Vapnik [7]. SVM is an important means to overcome the "dimension curse", over-fitting phenomenon and so on, mainly used in classification and regression problems. It established a model of sensor fault detection and diagnosis based on SVM. Early fault is detected for the sensor system by using SVR, and further identification of fault types is using SVC. The main idea of SVM is constructing a classification function to classify the samples for the training set. Assuming that, the training set is $T=\left\{\left(x_{1}, y_{1}\right),\left(x_{2}, y_{2}\right), \cdots,\left(x_{l}, y_{l}\right)\right\} \in(X \times Y)^{l}$. Where, $X$ denotes the input space, $Y$ denotes the output space. Each point $x_{i}$ in input space is composed by $n$ attributes, which can be described as $x_{i} \in X=R^{n}$. Each $y_{i}$ in the output space is the classification identification corresponding each $x$, described as $y_{i} \in Y=\{-1,1\}, i=1, \cdots, l$. Finding a real valued function $g(x)$ of the $R^{n}$, to construct the classification function $f(x)=\operatorname{sgn}(g(x))$ to infer any $x$ corresponding $y$. When the samples of the training set is linearly separable, existing only one standard hyperplane $(\omega \cdot x)+b=0$, When $(\omega \cdot x)+b \geq 1, y_{i}=1$; when $(\omega \cdot x)+b \leq-1, y_{i}=-1$. The two formulas merge into $y_{i}\left(\omega \cdot x_{i}+b\right) \geq 1$, which satisfy $y_{i}\left(\omega \cdot x_{i}+b\right)=1$ 
established $x_{i}$ called support vector.

For the linearly separable case, the support vector plays a role only when the classification hyperplane has been established, usually accounting for only a small part of the sample set, so that SVM having sparsity. The interval of the sample point of $y_{i}=1$ and standard hyperplane is $\min _{y_{i}=1} \frac{\left|\left(\omega \cdot x_{i}\right)+b\right|}{\|\omega\|}=\frac{1}{\|\omega\|}$, and then the support vector interval is $\frac{2}{\|\omega\|}$. The optimal hyperplane means that the maximum $\frac{2}{\|\omega\|}$. So to find the optimal hyperplane problem can be transformed into the following two quadratic programming problems.

$$
\begin{array}{ll}
\min & \frac{1}{2}\|\omega\|^{2} \\
\text { s.t. } & y_{i}\left(\left(\omega \cdot x_{i}\right)+b\right) \geq 1 \quad i=1, \cdots, l
\end{array}
$$

When the two types of samples in training set $T$ can be linearly separable, in addition to the general support vector distributes in two classification boundary of $y_{i}\left(\omega \cdot x_{i}+b\right)=1$, all the rest of the sample points are distributed in the classification boundary outside. The structured hyperplane is called margin hyperplane. When the two types of sample training set $T$ cannot be linearly separable, which allows not meet the constraints of the sample points, still can partition by using the hyperplane. It is time to soften the interval, to structure the soft margin hyperplanes. Softening method is introducing slack variable $\xi_{i} \geq 0, \quad i=1, \cdots, l$ to get the "softening" constraints $y_{i}\left(\left(\omega \cdot x_{i}\right)+b\right) \geq 1-\xi_{i}, i=1, \cdots, l$. When $\xi_{i}$ is sufficiently large, the sample points always satisfy the above constraints. But also trying to avoid $\xi_{i}$ too large value, it is going to punish it in the objective function. Get the following quadratic programming.

$$
\begin{array}{ll}
\min & \frac{1}{2}\|\omega\|^{2}+C \sum_{i=1}^{l} \xi_{i}, \\
\text { s.t. } \quad y_{i}\left(\left(\omega \cdot x_{i}\right)+b\right) & \geq 1-\xi_{i}, \\
& \xi_{i} \geq 0, \quad i=1, \cdots, l .
\end{array}
$$

where, $C \geq 0$ is the penalty parameter, the Lagrange function is.

$$
\begin{aligned}
& L(\omega, b, \xi, \alpha, \gamma)=\frac{1}{2}\|\omega\|^{2}+C \sum_{i=1}^{l} \xi_{i}- \\
& \sum_{i=1}^{l} \alpha_{i}\left(y_{i}\left(\left(\omega \cdot x_{i}\right)+b\right)-1+\xi_{i}\right)-\sum_{i=1}^{l} \gamma_{i} \xi_{i}
\end{aligned}
$$

where, $\gamma_{i} \geq 0, \xi_{i}>0$.

When the training set $T$, the two kinds of sample point set overlap area is large, dealing with linear inseparable problem of linear SVC will not be able to classify the sample. SVC by introducing the transformation of $x \mapsto \varphi(x)$ from the input space $X$ to another higher dimensional Hilbert space $H$, the original input space $X$ training sets $T=\left\{\left(x_{1}, y_{1}\right),\left(x_{2}, y_{2}\right), \cdots,\left(x_{l}, y_{l}\right)\right\} \in(X \times Y)^{l}$ translate into new training set in the Hilbert space $H$.

$$
\begin{aligned}
\bar{T} & =\left\{\left(\bar{x}_{1}, y_{1}\right),\left(\bar{x}_{2}, y_{2}\right) \cdots\left(\bar{x}_{l}, y_{l}\right)\right\} \\
& =\left\{\left(\varphi\left(x_{1}\right), y_{1}\right),\left(\varphi\left(x_{2}\right), y_{2}\right),\left(\varphi\left(x_{l}\right), y_{l}\right)\right\}
\end{aligned}
$$

It is made the Hilbert space $H$ linear separable. The Hilbert space $H$ means the feature space. In order to rigidly divide the training set $\bar{T}$, the hyperplane $(\omega \cdot \varphi(x))+b=0$ is obtained through the space $H$. The original problem is transformed into quadratic programming as follows.

$$
\begin{aligned}
& \min \frac{1}{2}\|\omega\|^{2} \\
& \text { s.t. } \quad y_{i}\left(\left(\omega \cdot \varphi\left(x_{i}\right)\right)+b\right) \geq 1
\end{aligned}
$$

By introducing the kernel function $K$, $K\left(x_{i}, x_{j}\right)=\left(\varphi\left(x_{i}\right) \cdot \varphi\left(x_{j}\right)\right)$ is made to avoid the complex operation in high dimensional feature space. Commonly used polynomial kernel function is polynomial kernel function, the radial basis kernel function and sigmoid kernel function. Where, the polynomial kernel function is expressed as $k((x \cdot y)+\theta)^{d}$; radial basis kernel function is expressed as $k(x \cdot y)=\exp \left(-\frac{\|x-y\|^{2}}{s^{2}}\right) ;$ sigmoid kernel function is expressed as $k(x \cdot y)=\tanh (u(x \cdot y)-r)$.

By introducing the Lagrange multiplier and according to the duality theorem Wolfe [10] convert (5) to its dual problem.

$$
\begin{array}{ll}
\max _{\alpha} & -\frac{1}{2} \sum_{i=1}^{l} \sum_{j=1}^{l} y_{i} y_{j} \alpha_{i} \alpha_{j} K\left(x_{i} \cdot x_{j}\right)+\sum_{i=1}^{l} \alpha_{i} \\
\text { s.t. } \quad & \sum_{i=1}^{l} y_{i} \alpha_{i}=0 \\
& \alpha_{i} \geq 0, i=1, \cdots, l
\end{array}
$$

When mapped the training set in the high dimensional space $H$ cannot be rigidly divided, need to soften of constraint conditions, it is time to introduce the penalty factor $C$.

$$
\begin{array}{ll}
\max _{\alpha} & -\frac{1}{2} \sum_{i=1}^{l} \sum_{j=1}^{l} y_{i} y_{j} \alpha_{i} \alpha_{j} K\left(x_{i} \cdot x_{j}\right)+\sum_{i=1}^{l} \alpha_{i} \\
\text { s.t. } & \sum_{i=1}^{l} y_{i} \alpha_{i}=0 \\
& 0 \leq \alpha_{i} \leq C, i=1, \cdots, l
\end{array}
$$


The optimal solution $\alpha^{*}=\left(\alpha_{1}^{*}, \cdots \alpha_{l}^{*}\right)^{T}$ is obtained by solving (6), $b^{*}=y_{i}-\sum_{i=1}^{l} y_{i} a_{i}^{*} K\left(x_{i}, x_{j}\right)$ is calculated by choosing the positive component $\alpha^{*}$ which is restrained by $0<\alpha_{j}^{*}<C$, to construct the decision function $g(x)=\sum_{i=1}^{l} y_{i} \alpha_{i}^{*} K\left(x \cdot x_{j}\right)+b^{*}$. The final classification function is constructed as follows.

$$
f(x)=\operatorname{sgn}\left(\sum_{i=1}^{l} y_{i} \alpha_{i}^{*} K\left(x \cdot x_{j}\right)+b^{*}\right)
$$

The unknown samples can be classified.

Generalized SVM to estimate real function by learning the samples, it is called the SVM regression problem [11]. SVR is widely used in the regression and the function approximation problem. Considering an independent identically distributed sample point $T=\left\{\left(x_{1}, y_{1}\right),\left(x_{2}, y_{2}\right), \cdots,\left(x_{l}, y_{l}\right)\right\} \in(X \times Y)^{l} \quad$, Where, $x_{i} \in X=R^{n}, y_{i} \in Y=\{-1,1\}$. Search function $y=f(x)$ to infer any $x$ corresponding $y$ value, and makes the empirical risk minimum.

In order to improve the robustness of the support vector regression, the insensitive loss function $\varepsilon$ has been introduced as follows.

$$
L_{\varepsilon}\left(f(x), y_{i}\right)=\left\{\begin{array}{cc}
\left|f(x)-y_{i}\right|-\varepsilon & \left|f(x)-y_{i}\right| \geq \varepsilon \\
0 & \text { else }
\end{array}\right.
$$

If the absolute value's deviation between the estimator output $f(x)$ and the desired output $y_{i}$ is less than $\mathcal{E}$, it equals to the zero; otherwise it equals to the deviation of the absolute value minus $\mathcal{E}$. The insensitive function can ensure the existence of the global minimum solution and the reliable optimization of generalization, having again with a subset of training set to express the computational advantage, so to improve the stability and generalization ability of the algorithm.

For a given training set $T$, the appropriate precision parameter $\varepsilon>0$, penalty parameter $C>0$ and suitable kernel function $K\left(x, x^{\prime}\right)$ have been chosen. Structuring and solving optimized problems as follows.

$$
\begin{aligned}
\min _{\alpha^{(*)} \in R^{2 l}} & \frac{1}{2} \sum_{i, j=1}^{l}\left(\alpha_{i}^{*}-\alpha_{i}\right)\left(\alpha_{j}^{*}-\alpha_{j}\right) K\left(x_{i}, x_{j}\right)+ \\
\varepsilon & \sum_{i=1}^{l}\left(\alpha_{i}^{*}-\alpha_{i}\right)-\sum_{i=1}^{l} y_{i}\left(\alpha_{i}^{*}-\alpha_{i}\right) \\
\text { s.t. } \quad & \sum_{i=1}^{l}\left(\alpha_{i}^{*}-\alpha_{i}\right)=0 \\
& 0 \leq \alpha_{i}, \quad \alpha_{i}^{*} \leq \frac{C}{l}, \quad i=1, \cdots, l
\end{aligned}
$$

The optimal solution $\bar{\alpha}^{(*)}=\left(\bar{\alpha}_{1}, \bar{\alpha}_{1}^{*}, \cdots, \bar{\alpha}_{l}, \bar{\alpha}_{l}^{*}\right)^{T}$ is obtained by (8), choosing $\bar{\alpha}^{(*)}$ positive component of $\bar{\alpha}_{j}>0$, calculating $\bar{b}=y_{i}-\sum_{i=1}^{l}\left(\alpha_{i}^{*}-\alpha_{i}\right) K\left(x_{i}, x_{j}\right)+\mathcal{E}, \quad$ so to construct the decision function as follows.

$$
y=\sum_{i=1}^{l}\left(\alpha_{i}^{*}-\alpha_{i}\right) K\left(x_{i}, x_{j}\right)+\bar{b}
$$

The unknown samples can be predicted.

\section{The SVM Parameters Optimization BASED ON CLONAL SELECTION ALGORITHM}

When the distribution area of two kinds of sample points in the input space was serious overlapped. It can make the distribution area of the sample points which are reflected to each class of feature space more concentrated by selecting the proper kernel function and parameters. So as to reduce the combination of the distribution area of two kinds of sample points, and strengthen two kinds of sample set in the feature space "Linear separability" to achieve the purpose of improving the precision of classification and generalization performance. However, while electing nuclear function and parameters, using different kernel functions for the same experimental data, the accuracy tends to be varying widely. Even for the same kernel function, the selection of parameters is different; the precision of classification will have bigger difference. In the process of practical application, through the simulation for many times to find the suitable kernel function, and determine the best parameters.

It presents a method that SVM parameters optimization based on genetic algorithm in [12], the selection of SVM parameters is regarded as a function optimization problem, utilizing the global optimization ability of genetic algorithm is to optimize the parameters of SVM. To some extent, although this method can optimize the performance of SVM, the genetic algorithm exist the disadvantage that the speed of convergence is slow, and easy to fall into the defect of local optimal solution.

Biological immune recognition method of the biological immune system can use the relatively limited number of antibodies to identify an almost infinite number of antigen in a short period of time [13]. The genetic recombination, affinity maturation and receptor editing mechanism of the clonal selection can well describe the formation of problem solving ability. Basic thought of the clonal selection is that the cells which are able to identify the antigen cells are augmenting, and these cells are selected and preserved. The cells which cannot identify the antigen cells are not selected, and not augmenting. CSA can improve the rate of convergence, at the same time, keep the population diversity. What's more, CSA can effectively overcome the problems such as premature convergence; it is suitable for processing optimization problem. When using the clonal selection algorithm for SVM parameters optimization, it will be the selection of optimization parameters corresponding antigen and feasible solutions corresponding antibody and feasible solution corresponding the optimal solution of approximation degree of affinity. As a result, the optimization process of the SVM parameter optimization problem can be with the immune system to identify antigens and the process of antibodies evolution. To abstract the evolutionary in 
biological immune response chain as the optimization process for the evolution of the math [14]. (Original antibody group - selection to clone amplification - high frequency mutation - reselect the best antibodies - the new antibody group). Fig. 2 shows the detail block diagram of CSA optimization algorithm.

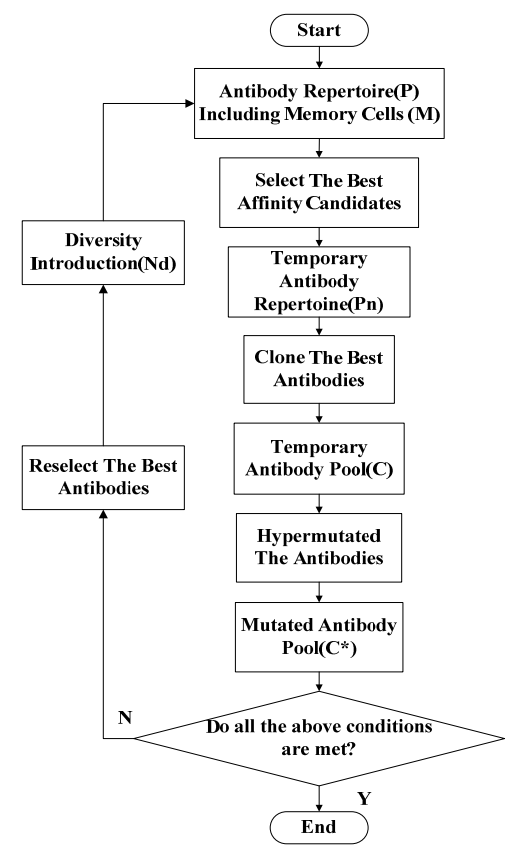

Fig. 2. Block diagram of parameters optimization by clonal selection algorithm.

First, randomly generated antibodies repertoire $P$, it is the sum of memory antibody cells $(M)$ and the temporary antibody repertoire $\left(P_{r}\right),\left(P=P_{r}+M\right)$. Calculating the affinity of antibodies $A b_{-}$aff, and choose the antibodies of high affinity individuals to constitute a temporary group of $P_{n}$. Second, $N$ of the best individual in the group $P_{n}$ are cloning to generate the temporary clone pool $C . N$ of the antibodies which are selected will be cloned, the higher the antibody affinity, the higher the number of antibody clone $\left(N_{c}\right)$. Where, $N_{c}$ is described as $N_{c}=\sum_{i=1}^{n} \operatorname{round}\left(\frac{\beta . N}{i}\right)$ to determine the number of antibody clone. Third, the operation of high frequency mutation to generate a mature antibody group $C^{*}$, where, the mutation probability is inversely proportional to the antibody affinity. Finally, recalculating the mutated antibody affinity with higher affinity antibodies is to replace the original, update memory antibodies $M$, $d$ of the low affinity antibodies to be replaced, so as to maintain the diversity of antibody.

\section{The EMB System Sensor FAult Detection AND DiAGNOSIS BASED ON THE MODIFIED CSA-SVM}

Common sensor faults mainly include stuck fault, constant gain fault and constant bias fault. Among them, when stuck fault happens, the output of sensor is a certain value, which can be described as $y_{\text {out }}(t)=a \neq y_{\text {in }}(t)$. Sensor constant gain fault will make the output amplitude change, which can be described by $y_{\text {out }}(t)=\beta y_{\text {in }}(t)$.The output of sensor which has constant bias fault lags behind normal output, which can be described by $y_{\text {out }}(t)=y_{\text {in }}(t)+\Delta$.

Sensor fault detection and diagnosis of EMB system is based on SVM classification and regression prediction method in this paper. SVR is used for regression prediction of three sensors fault in EMB. Through the residual errors between the prediction model outputs and the actual system outputs, the fault can be detected, and the fault information is outputted to support vector classification to further distinguish the type of sensor fault. The architecture of EMB sensor fault detection and diagnosis is shown in Fig. 3.

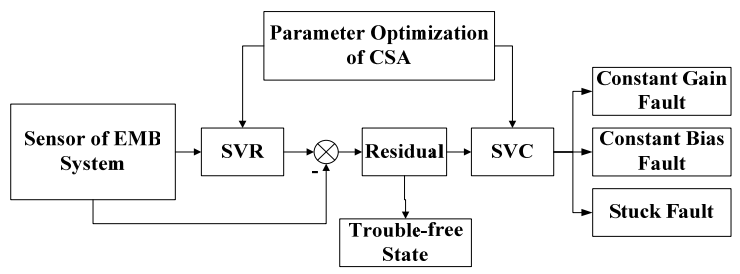

Fig. 3. EMB sensor fault detection and diagnosis model.

The specific process of EMB sensor fault detection and diagnosis based on CSA-SVM is divided into early prediction and category recognition of fault. In period of early prediction, first based on motor model of EMB system, mathematical models of current and speed sensor are established, respectively. And pressure sensor model is established in view of the brake actuator structure. Through acquisition of the sensor output data, training sample set is established. Then considering the punishment and kernel parameters of SVM, CSA is used to optimize SVM. Specific optimization algorithm is determining the parameters of CSA, including the evolution algebra, crossover probability and mutation probability. Using CSA randomly generated initial antibodies, and selecting the first $N$ antibody, then decoding it for the kernel and punishment parameter. The K-fold cross-validation method is used to calculate the correct classification rate of the antibody. On the basis of the rate of correct classification to test sample by antibodies in antibody repertoire, antibodies are dealt with selective clone, crossover and mutation operation [15]. Output optimal affinity antibody as the parameters of SVM, and draw the corresponding support vector and the classification hyper plane coefficient. In the end, put the penalty parameter $C$ and RBF kernel parameters from clonal selection optimization in SVM. According to the decision function (9), calculate the new sample data of EMB system sensor fault. Then to generate residuals by comparing estimated output values of sensor from SVR. And the actual output values of EMB for early sensor fault prediction.

In fault category recognition, input the EMB fault sample data to CSA-SVM. According to the decision function (7), the specific fault types will be identified based on the calculation and process for fault sample data.

\section{Simulation EXPERIMENT AND RESUlt ANALYSiS}

In order to verify the validity of the sensors fault detection and diagnosis model proposed in this study, the mathematical modeling and simulation of EMB system sensor faults such 
as stuck , constant gain and constant bias faults are carried out based on MATLAB.

Collecting the normal and fault signal of EMB sensors as the training sample of SVM, at the same time, to optimize the punishment factor and radial basis kernel parameters for SVM by using the CSA. Where, the parameters of CSA as shown in Table I.

TABLE I: PARAMETERS OF ClONE SELECTION AlgORITHM

\begin{tabular}{c|c}
\hline \hline Parameter & Value \\
\hline Antibodies scale & 20 \\
\hline encoding length & 10 \\
\hline encoding length $(\mathrm{Pc})$ & 0.2 \\
\hline Mutation rate $(\mathrm{Pm})$ & 0.01 \\
\hline Selected individual number $(\mathrm{m})$ & 12 \\
\hline Proportional control factor & 0.2 \\
\hline Antibody concentration threshold & 0.6 \\
\hline Evolution generation & 100 \\
\hline \hline
\end{tabular}

Put the signal of the sensor mathematical model which is proposed in Section II into the support vector regression model to achieve the estimated signal based on CSA-SVM.

Compare the result from the CSA-SVM fault detection model with the traditional SVM fault detection model to take simulation. The status output simulation of the sensor trouble-free results is shown in Fig. 4.
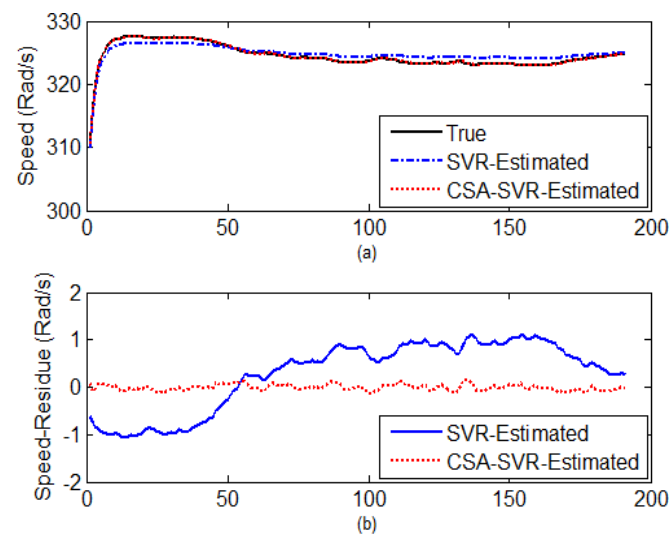

Fig. 4. Sensor trouble-free status simulation results. (a) The comparison of the sensor actual output, the traditional SVR and the modified CSA-SVR predicted output; (b) the comparison of the traditional SVR and the modified CSA-SVR residual value.

Fig. 4 (a) shows the comparison of the sensor actual output, the SVR and the CSA-SVR predicted output. Where, the solid line is the sensor actual output, the long dotted line is the estimated output by traditional SVR model, the short dash line is the estimated output by CSA-SVR model. Fig. 4 (b) shows the comparison of the SVR and the CSA-SVR residual value. Where, the solid line is the residual value between sensor actual output and traditional SVR estimated, the dotted line is the residual value between sensor actual output and modified CSA-SVR estimated. From the simulation results, we can see that the SVM can fit the output of sensor under the trouble-free status. Through analyzing the test results, it proves that precision of the regression fitting model based on modified CSA-SVR increased $62.5 \%$ to the traditional SVR, and the output of sensor can be more predict accurately.

Fig. 5 shows the simulation results of the EMB system sensor stuck fault signal detection and estimation.
Fig. 5 (a) shows the comparison of the sensor actual output, the SVR and the CSA-SVR predicted output. While the sensor is stuck fault, the system output value remains in the current value, and keeps stable afterwards. Fig.5 (b) shows the comparison of the SVR and the CSA-SVR residual value. Two kinds of SVR estimated residual occurs obviously jumping, and hold steady residual value after failure.

Fig. 6 shows the simulation results of the EMB system sensor constant gain fault signal detection and estimation.
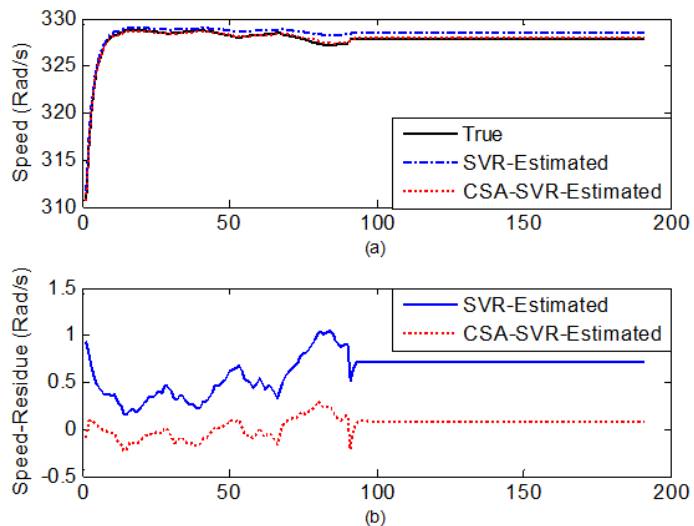

Fig. 5. Sensor stuck fault signal detection and estimation. (a) The comparison of the sensor actual output, the traditional SVR and the modified CSA-SVR predicted output; (b) the comparison of the traditional SVR and the modified CSA-SVR residual value.
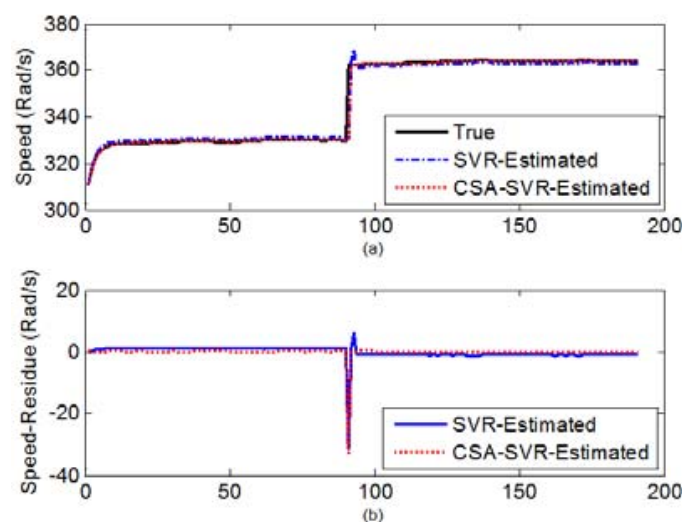

Fig. 6. Sensor constant gain fault signal detection and estimation. (a) The comparison of the sensor actual output, the traditional SVR and the modified CSA-SVR predicted output; (b) the comparison of the traditional SVR and the modified CSA - SVR residual value.
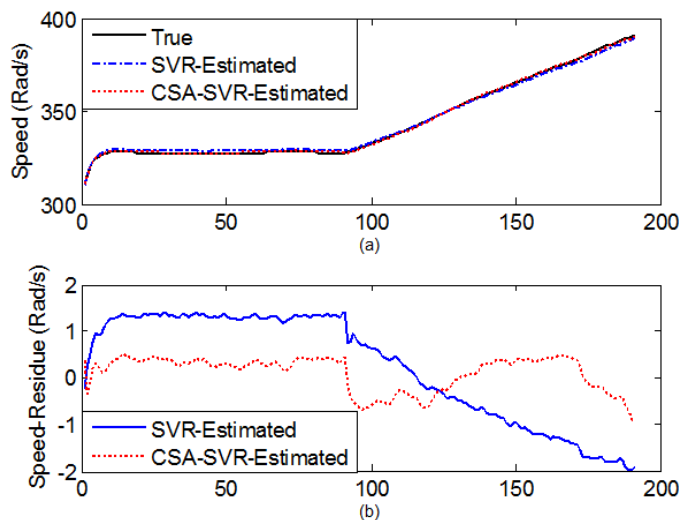

Fig. 7. Sensor constant bias fault signal detection and estimation.(a) The comparison of the sensor actual output, the traditional SVR and the modified CSA-SVR predicted output; (b) the comparison of the traditional SVR and the modified CSA-SVR residual value.

Fig. 6 (a) shows the comparison of the sensor actual output, the SVR and the CSA-SVR predicted output. It can be seen 
from the simulation results that while the sensors constant gain fault, the output of the system suddenly alpha times to the normal state, and then keeps stable afterwards. Fig. 6 (b) shows the comparison of the SVR and the CSA-SVR residual value. The simulation results show that two kinds of SVR system that estimates residual can detect the fault because of the abrupt change when the fault occurred.

Fig. 7 shows the simulation results of the EMB system sensor constant bias fault signal detection and estimation.

Where, Fig. 7 (a) shows the comparison of the sensor actual output, the SVR and the CSA-SVR predicted output. The simulation results show that great deviation appeared when the sensor constant bias fault occurred, and affected the normal operation of the sensor. Fig. 7 (b) shows the comparison of the SVR and the CSA-SVR residual value. It shows that when the sensor constant bias fault happened, the estimated residual change obviously so that the fault can be detected Immediate and accurately.

Put the sensor fault signal into the SVM classifier, the specific sensor fault types can be further identified. Using the clonal selection algorithm is to optimize the parameters of the SVM classifier, and regard the traditional SVM as a comparison to the experimental simulation.

Fig. 8 and Fig. 9 show the three kinds of sensor fault diagnosis results of EMB system towards the traditional SVM and CSA-SVM, respectively.

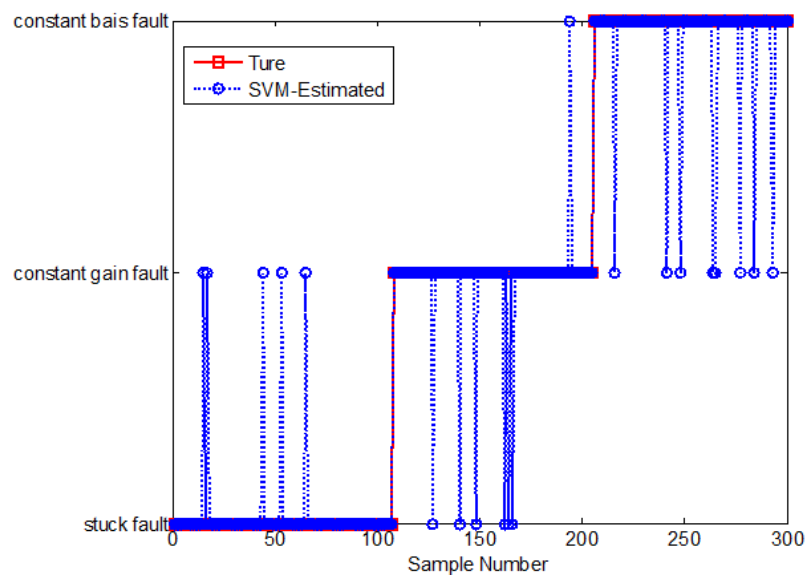

Fig. 8. SVM sensor fault diagnosis simulation diagram

TABLE II: THE COMPARISON OF THE SENSOR FAUlt DiAGNOSTIC RATE

\begin{tabular}{|c|c|c|c|}
\hline $\begin{array}{l}\text { Sensor } \\
\text { Type }\end{array}$ & $\begin{array}{l}\text { Fault } \\
\text { Type }\end{array}$ & $\begin{array}{c}\text { SVM } \\
(\%)\end{array}$ & $\begin{array}{c}\text { CSA-SVM } \\
(\%)\end{array}$ \\
\hline \multirow{3}{*}{$\begin{array}{l}\text { Current } \\
\text { Sensor }\end{array}$} & Stuck & 0.8942 & 0.9608 \\
\hline & $\begin{array}{c}\text { Constant } \\
\text { Gain }\end{array}$ & 0.8585 & 0.9510 \\
\hline & $\begin{array}{c}\text { Constant } \\
\text { Bias }\end{array}$ & 0.9356 & 0.9571 \\
\hline \multirow{3}{*}{$\begin{array}{l}\text { Speed } \\
\text { Sensor }\end{array}$} & Stuck & 0.8846 & 1.0000 \\
\hline & $\begin{array}{c}\text { Constant } \\
\text { Gain }\end{array}$ & 0.8762 & 0.9310 \\
\hline & $\begin{array}{c}\text { Constant } \\
\text { Bias }\end{array}$ & 0.9057 & 0.9798 \\
\hline \multirow{3}{*}{$\begin{array}{l}\text { Pressure } \\
\text { Sensor }\end{array}$} & Stuck & 0.9327 & 0.9928 \\
\hline & $\begin{array}{l}\text { Constant } \\
\text { Gain }\end{array}$ & 0.7830 & 0.9302 \\
\hline & $\begin{array}{c}\text { Constant } \\
\text { Bias }\end{array}$ & 0.8360 & 1.0000 \\
\hline
\end{tabular}

where, the rectangular solid line is the real value of the fault type, the circular dotted line is the estimated value of fault classification towards SVM. The simulation results showed that the three kinds of fault of sensors can be classified by
SVM. Compared with traditional SVM, the modified CSA-SVM has a more accurate and more stable identification function towards sensor faults. The traditional SVM model compared with modified CSA-SVM for sensor fault diagnosis results have been shown in Table II.

The Table II shows that the average value of the traditional SVM sensor fault diagnosis model classification accuracy is $87.8 \%$, and the accuracy of the fault classification based on the modified CSA-SVM fault diagnosis model reaches $96.7 \%$. Analyzing the simulation results by repeating the experiment for many times, the accuracy of fault classification modified CSA-SVM model can be increased by $10 \%$ than the traditional SVM.

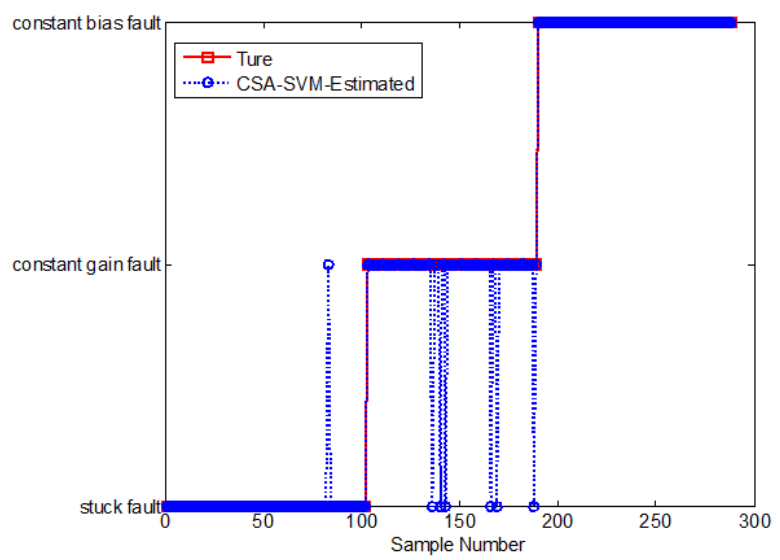

Fig. 9. CSA-SVM sensor fault diagnosis simulation diagram.

\section{CONCLUSION}

This article researches on fault detection and diagnosis which is aimed at the current, rotational speed and the pressure sensor of the EMB system. It is proposed a model of the fault detection and diagnosis based on SVM. At the same time, the SVM parameter optimization model has been proposed based on CSA, which is aimed at a big problem of the fault classification accuracy of SVM and the stability of the fault detection which are greatly influenced. Firstly using the CSA-SVR is to give early prediction to the system, comparing the forecast output with the actual output of the transducer to generate the residuals. By detecting the change of the residuals is to give early prediction to the failure, and using SVC is to identify the fault type of the sensors farther. Simulation experiments show that the SVM model for sensor fault detection and diagnosis based on the clonal selection algorithm optimization can give the real-time detection and diagnosis to the sensor fault, at the same time, comparing the CSA-SVM with the traditional SVM, it can improve the reliability of $62.5 \%$ in fault detection, and improve the accuracy of fault classification by $10 \%$ in the identification of fault. It is proposed that the modified CSA-SVM model can accurately detect the fault type of the fault sensor to lay a foundation for the subsequent intelligent fault tolerant control technology of EMB system.

\section{REFERENCES}

[1] J. K. Ahn, K. H. Jung, D. H. Kim, H. B. Jin, H. S. Kim, and S. H Hwang, "Analysis of a regenerative braking system for hybrid electric vehicles using an electro-mechanical brake," International Journal of Automotive Technology, vol. 10, pp. 229-234, Aug. 2009. 
[2] J. Böhm, S. Stölzl, and P. Willimowski, "Electromechanical brake system," Patent, vol. 345, no. 225, Feb. 5, 2002.

[3] R. H. Chen and J. L. Speyer, "Robust multiple - fault detection filter," International Journal of Robust and Nonlinear Control, vol. 12, pp. 675-696, Dec. 2002.

[4] S. R. Naidu, E. Zafiriou, and T. J. McAvoy, "Use of neural networks for sensor failure detection in a control system," IEEE Control Systems Magazine, vol. 10, pp. 49-55, May, 1990.

[5] Z. Du, X. Jin, and Y. Yang, "Fault diagnosis for temperature, flow rate and pressure sensors in VAV systems using wavelet neural network," Applied energy, vol. 86, pp. 1624-1631, Sep. 2009.

[6] J. Wu, Y. Zhao, and R. He, "Fault detection and diagnosis of EMB sensor system based on SVR," Engineering and Technology Journal of Jilin University, vol. 43, pp. 1178-1183, May, 2013.

[7] W. Hwang, K. Han, and K. Huh, "Fault detection and diagnosis of the electromechanical brake based on observer and parity space," International Journal of Automotive technology, vol. 13, pp. 845-851, Aug. 2012.

[8] R. He, J. Li, Y. D. Li, C. C. Huang, and Q. Wei, "Fault detection approach to EMB sensors based on dedicated observers," in Proc. 2011 International Conference on Electric Information and Control Engineering, pp. 3266-3269, April, 2011.

[9] V. Vapnik, The Nature of Statistical Learning Theory, New York, Springer-Verlag, 1995.

[10] Z. Q. Luo and S. Z. Zhang, "On extensions of the Frank-Wolfe theorems," Computational Optimization and Applications, vol. 13, pp. 87-110, April 1999.

[11] A. Widodo and B. S. Yang, "Support vector machine in machine condition monitoring and fault diagnosis," Mechanical Systems and Signal Processing, vol. 21, pp. 2560-2574, Jan. 2007.

[12] S. Fei and X. Zhang, "Fault diagnosis of power transformer based on support vector machine with genetic algorithm," Expert Systems with Applications, vol. 36, pp. 11352-11357, Oct. 2009.
[13] D. L. N. Castro and F. J. V. Zuben, "Learning and optimization Using the Clonal Selection Principle," IEEE Transactions on Evolutionary Computation, vol. 6, pp. 239-251, Jun. 2002.

[14] L. H. Song, W. J. Chang, and C. G. Jiu, "Analog circuit fault diagnosis based on SVM optimized by CSA," Journal of Electronic Measurement and Instrument, vol. 24, pp. 1132-1136, Oct. 2010.

[15] T. F. Lee, M. Y. Cho, C. S. Shieh et al., "Diagnosis of incipient fault of power transformers using SVM with clonal selection algorithms optimization," Foundations of Intelligent Systems, Springer Berlin Heidelberg, vol. 4203, pp. 580-590, Sep. 2006.

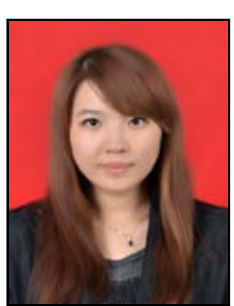

Z. J. Yu was born in 1990, Baishan, China. Currently she is still pursuing her master degree in the School of Yanbian University, Yanji, China.

She completed bachelor degree in electronics engineering and technology, Jilin, Yanji, in the field of electronics and telecommunication, in 2013.

Her research interests include fault diagnosis and fault-tolerant control.

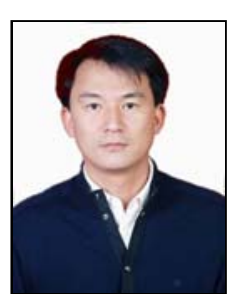

Y. N. Xu was born at Jilin province of China. Currently, he is an associate professor of the Division of Electronic and Communication Engineering of Yanbian University, Yanji, China. $\mathrm{He}$ received the $\mathrm{Ph} . \mathrm{D}$. degree in electrical engineering from the Chonbuk National University, Korea, in 2009.

His research interests include the in-vehicle networks and automobile electronic control. 